\title{
Rank aggregation in cyclic sequences
}

\author{
Javier Alcaraz ${ }^{1}, \quad$ Eva M. García-Nové ${ }^{1}, \quad$ Mercedes Landete ${ }^{1 *}$, \\ Juan F. Monge ${ }^{1}$, Justo Puerto ${ }^{2}$ \\ ${ }^{1}$ Department of Statistics, Mathematics and Computer Science, \\ Center of Operations Research, \\ Miguel Hernández University of Elche, Spain \\ ${ }^{2}$ IMUS. University of Seville, Spain.
}

\begin{abstract}
In this paper we propose the problem of finding the cyclic sequence which best represents a set of cyclic sequences. Given a set of elements and a precedence cost matrix we look for the cyclic sequence of the elements which is at minimum distance from all the ranks when the permutation metric distance is the Kendall Tau distance. In other words, the problem consists of finding a robust cyclic rank with respect to a set of elements. This problem originates from the Rank Aggregation Problem for combining different linear ranks of elements. Later we define a probability measure based on dissimilarity between cyclic sequences based on the Kendall Tau distance. Next, we also introduce the problem of finding the cyclic sequence with minimum expected cost with respect to that probability measure. Finally, we establish certain relationships among some classical problems and the new problems that we have proposed.
\end{abstract}

Keywords: Linear Ordering Problem, Rank Aggregation Problem.

\section{Introduction}

There is a vast number of combinatorial problems which look for the best permutation of a set of elements assuming a certain criterion. If the optimal permutation is the one which minimizes the cost of the path induced by the permutation we have the Linear Ordering Problem (LOP) ([8], [10]) and when the optimal permutation is the closest to a given set of permutations and the distances among permutations are measured with the Kendall Tau distance, the problem results in the Rank Aggregation Problem (RAP) (see [1], [4], [5] and [11] for a description and evolution of the RAP and its applications). These two problems are closely related and it can be shown that the RAP reduces to the LOP under a suitable transformation. In this paper we

${ }^{*}$ Corresponding author - email: landete@umh.es; Tel: +34 966658718; Fax: +34966658715 


\begin{tabular}{r|rrrrrr} 
& 123 & 132 & 213 & 231 & 312 & 321 \\
\hline 123 & 0 & 1 & 1 & 2 & 2 & 3 \\
132 & 1 & 0 & 2 & 3 & 1 & 2 \\
213 & 1 & 2 & 0 & 1 & 3 & 2 \\
231 & 2 & 3 & 1 & 0 & 2 & 1 \\
312 & 2 & 1 & 3 & 2 & 0 & 1 \\
321 & 3 & 2 & 2 & 1 & 1 & 0
\end{tabular}

Table 1: Kendall Tau distance matrix, $\Delta_{6 \times 6}$.

propose to afford these problems when the set of elements must be ranked in a cyclic way: for instance when the elements are slices in a pie chart or nodes in a routing problem. The reader may observe that the above problem is related to the so-called Target Visitation Problem (TVP) ([6] and [7]). Later, we will show the relationships between them.

In this paper we introduce the Cyclic Rank Aggregation Problem (CRAP) and a variant of it, that we call the Permutated Asymmetric Traveling Salesman Problem ( $\rho$ ATSP). The Cyclic Rank Aggregation Problem consists in finding the cyclic sequence which is at minimum distance from a given set of cyclic sequences by measuring distances with the Kendall Tau metric. The Permutated Asymmetric Traveling Salesman Problem consists in finding the cyclic sequence with smaller expected cost. The applications of the Target Visitation Problem, as for example environment assessment, combat search, rescue and disaster relief $([6])$ and applications to the delivery of emergency supplies ([3]) can also be shown as examples of application of the Cyclic Rank Aggregation Problem and of the Permutated Asymmetric Traveling Salesman Problem. This paper also shows that an optimal solution of the $\rho$ ATSP is an optimal solution of CRAP but in reverse order.

The main contribution of this paper is a compact formulation for the Cyclic Rank Aggregation Problem and the proof of equivalence of the Cyclic Rank Aggregation Problem and the Linear Ordering Problem. The paper is organized as follows. In Sections 2 and 3 we introduce the CRAP and the $\rho$ ATSP respectively and we propose compact formulations for them. Section 5 is devoted to establish the relationships among the LOP, RAP, CRAP and $\rho$ ATSP.

\section{The Cyclic Rank Aggregation problem}

Let $G=(V, A)$ be a complete directed graph with arc weight $c_{i j}$ for each pair $i, j \in V$ and $V=\{1, \ldots, n\}$. Let $S$ be the full set of permutations in $V$ and $\sigma_{i}$ a permutation. We can define the distance $d\left(\sigma_{1}, \sigma_{2}\right)$ like the number or pairwise disagreements between the two permutations $\sigma_{1}$ and $\sigma_{2}$. This distance is known as Kendall tau distance.

\section{Definition 1}

The Kendall Tau distance between two permutations $\sigma_{1}$ and $\sigma_{2}$ is given by:

$$
d\left(\sigma_{1}, \sigma_{2}\right)=\left|\left\{(i, j): i<j,\left(\left(\sigma_{1}(i)<\sigma_{1}(j) \wedge \sigma_{2}(i)>\sigma_{2}(j)\right) \vee\left(\sigma_{1}(i)>\sigma_{1}(j) \wedge \sigma_{2}(i)<\sigma_{2}(j)\right)\right)\right\}\right|
$$


where, $\sigma_{1}(i)$ and $\sigma_{2}(i)$ are the position of the element $i$ in $\sigma_{1}$ and $\sigma_{2}$ respectively.

The Kendall Tau distance is a permutations metric that counts the number of pairwise disagreements between two ranking lists. The larger the distance is, the more different the permutations are. For instance, if we had four elements, the distance to the permutation 1234 from 1243, 1342 and 1432 will be 1, 2 and 3 respectively. Kendall Tau distances between all the permutations of four elements are shown in Table 1. In the following, we call $\Delta_{n \times n}=\left(\delta_{i j}\right)$ the Kendall distance matrix for $n$ elements.

We can define a cyclic ordering of a finite set $V$ to be a permutation $\sigma \in S$ with exactly one orbit. Cyclic orderings split the set of linear orderings $S$ into a set of equivalence classes. Similar to Definition 1, we can define the Kendall Tau distance between two cyclic sequences as the Kendall Tau distance between the representative cyclic sequences of each equivalent class. We denote by $R$ the set of equivalence class induced by $S$, when $S$ is the set of all permutations in $V$ (any $\rho \in R$ represents a hamiltonian tour in $V$ ). We denote by $C(r)$ the aggregation weights of the cyclic sequence $r \in R$ and we denote by $\rho \in R$ one element of $R$.

We define the Cyclic Rank Aggregation problem as the problem to find the cyclic sequence closest to all cyclic sequences in the complete directed graph $G=(V, A)$ :

$$
\text { (CRAP) } \arg \min _{\rho} \sum_{r \in R} C(r) d(\rho, r) .
$$

In order to exactly solve the CRAP we propose a compact formulation. We define a family of binary variables, called $z$-family:

$$
z_{i j}=\left\{\begin{array}{rr}
1 & \text { if } j \text { is visited before } i \\
0 & \text { otherwise }
\end{array}\right.
$$

for all $i, j \in V i \neq j$.

If $V=\{1,2,3,4\}$, the cyclic sequences 1234 and 1342 will be associated to the solutions

$$
z_{i j}=\left(\begin{array}{cccc}
- & 0 & 0 & 0 \\
1 & - & 0 & 0 \\
1 & 1 & - & 0 \\
1 & 1 & 1 & -
\end{array}\right) \text { and } z_{i j}=\left(\begin{array}{cccc}
- & 0 & 0 & 0 \\
1 & - & 1 & 1 \\
1 & 0 & - & 0 \\
1 & 0 & 1 & -
\end{array}\right)
$$

respectively.

The first contribution of this paper gives an explicit expression for the objective function of CRAP. Later, we shall substantially simplify it by using properties of the $z$-variables. 


\section{Proposition 1}

The objective function of CRAP is

$$
\begin{array}{r}
\sum_{i, j \in V-\{1\}: i \neq j} c_{i j}\left((n-2) ! z_{i j}+\sum_{k, t \in V-\{i, j, 1\}: k<t} \frac{(n-2) !}{2}\left(z_{k t}+z_{t k}\right)+\right. \\
\left.\sum_{k \in V: k \neq i, j, 1} \frac{(n-2) !}{2}\left(z_{k i}+z_{i k}+z_{j k}+z_{k j}\right)\right)+ \\
\sum_{j \in V-\{1\}} c_{1 j}\left((n-2) ! \sum_{k \in V-\{1, j\}} z_{j k}+\sum_{k, t \in V-\{j, 1\}: k<t} \frac{(n-2) !}{2}\left(z_{k t}+z_{t k}\right)\right)+ \\
\sum_{j \in V-\{1\}} c_{j 1}\left((n-2) ! \sum_{k \in V-\{1, j\}} z_{k j}+\sum_{k, t \in V-\{j, 1\}: k<t} \frac{(n-2) !}{2}\left(z_{k t}+z_{t k}\right)\right)
\end{array}
$$

The idea of the proof is to compute the number of times that each cost $c_{i j}$ appears in the objective function. For instance, consider a problem with $n=5$ in which the cheapest cyclic sequence is 12345 . Cost $c_{53}$ appears in permutations 12453, 12534, 14253, 14532, 15324 and 15342 which have disorders $2,2,3,5,4,5$. Thus, the coefficient of $c_{53}$ in the objective function is $21(2+2+3+5+4+5)$. And also $21=6 z_{53}+3\left(z_{32}+z_{42}+z_{52}+z_{43}+z_{54}\right)$, i.e., six times we add the disorder of visiting node 5 before node 3 , three times we add the disorder of visiting node 3 before node 2 and so on.

Proof of Proposition 1. The proof consists in computing the number of times that each $\operatorname{cost} c_{i j}$ appears in the objective function. Since all the cyclic sequences start at node 1 , we distinguish between $c_{i j}$ with $i, j \in V \backslash\{1\}, i \neq j$ and $c_{1 j}$ or $c_{j 1}$. We say that an ordered set of two nodes $\{i, j\}$ incurs in disorder if $j$ is visited before $i$ in the solution.

We distinguish three cases:

- $i, j \in V \backslash\{1\}, i \neq j . c_{i j}$ is added to the objective function when the arc $(i, j)$ belongs to the cyclic sequence and either $\{i, j\}$ incurs itself in disorder or any of the sets $\{k, i\},\{i, k\},\{k, j\},\{j, k\}$ for $k \in V-\{1, i, j\}$ or $\{k, t\},\{t, k\}$ for $k, t \in V-\{1, i, j\}, k \neq t$ incur in disorder. Therefore, we need to compute: (i) the number of permutations to which the arc $(i, j)$ belongs to; (ii) how many permutations among them visit $k$ before and after $i$ and before and after $j$ for all $k \in V-\{1, i, j\}$; (iii) how many permutations among them visit $k$ before $t$ and viceversa with $k, t \in V-\{1, i, j\}, k \neq t$.

- The arc $(i, j)$ belongs to $\{n-2\}$ ! permutations starting at 1 : there are $n-2$ positions for $i$ if it must be followed by $j$. Once $i$ and $j$ are assigned the other $n-3$ positions can be sorted anyhow, thus $\{n-2\} \times\{n-3\} !=\{n-2\}$ !. The first addend of the coefficient of $c_{i j}$ is $(n-2) ! z_{i j}$.

- By symmetry, in half of the $\{n-2\}$ ! permutations having arc $(i, j) k$ is visited before $t$ and in half of the permutations $t$ is visited after $k$ for $k, t \in V-\{1, i, j\}, k \neq t$. The 
second addend of the coefficient of $c_{i j}$ is

$$
\sum_{k, t \in V-\{i, j, 1\}: k<t} \frac{(n-2) !}{2}\left(z_{k t}+z_{t k}\right)
$$

- By symmetry, in half of the $\{n-2\}$ ! permutations having arc $(i, j), k$ is visited before $i$ and in half of the permutations $k$ is visited after $i$ for $k \in V-\{1, i, j\}$. Analogously, for the number of times that $k$ is visited before and after $j$. The third addend of the coefficient of $c_{i j}$ is

$$
\sum_{k \in V: k \neq i, j, 1} \frac{(n-2) !}{2}\left(z_{k i}+z_{i k}+z_{j k}+z_{k j}\right) .
$$

- $j \in V \backslash\{1\} . c_{1 j}$ is added to the objective function when the arc $(1, j)$ belongs to the cyclic sequence and any of the sets $\{j, k\}$ for $k \in V-\{1, j\}$ or $\{k, t\},\{t, k\}$ for $k, t \in V-\{1, j\}$, $k \neq t$ incur in disorder. It is similar to the case for $c_{i j}$ with $i>1$ but with two differences: (i) Since 1 is always the first node, the set $\{1, j\}$ never incurs itself in disorder; (ii) the number of permutations among those with the arc $(1, j)$ such that $k \in V-\{1, j\}$ is visited before $j$ is zero. Therefore, we need to compute the number of permutations to which the arc $(1, j)$ belongs and how many among them visit $k$ before $t$ and viceversa with $k, t \in V-\{1, i, j\}, k \neq t$. It is easy to observe that the number of permutations with the arc $(1, j)$ is $(n-2)$ ! and that half of them visit $k$ before $t$.

- $c_{j 1}$ for $j>1$. Analogous to the previous case.

Applying Proposition 1, we can formulate the CRAP as:

$$
\begin{array}{ll}
\text { (CRAP) min } & (2) \\
\text { s.t. } & z_{i j}+z_{j i}=1 \quad \forall i, j \in V: i<j \\
& z_{i j}-z_{i k}-z_{k j} \leq 0 \quad \forall i, j, k \in V: i \neq j, j \neq k, i \neq k \\
& z_{j 1}=1 \quad \forall j \in V-\{1\} \\
& z_{i j} \in\{0,1\} \quad \forall i, j \in V: i \neq j
\end{array}
$$

Constraint (3) translates the fact that any element $i$ is ranked before or after $j$ but both cases cannot occur simultaneously. It implies that both values $z_{j i}=0$ and $z_{i j}=1$ represent that element $j$ is ranked before element $i$. Constraint (4) describes the transitive relationship between the position of three elements in the permutation. The message is that if $j$ goes after $k$ and $k$ goes after $i$, (i.e., $z_{i k}=z_{k j}=0$ or $z_{k i}=z_{j k}=1$ ) then $j$ must go after $i$, i.e., $z_{j i}=1$ and $z_{i j}=0$. Any solution of the system (3), (4),(6) is a permutation of the elements of $V$ : all the nodes are relative sorted and transitivity holds. Indeed, the set of constraints $(3),(4),(6)$ is the set of constraints for the LOP. (5) forces node 1 to be the first node of any cyclic sequence. 
Since any solution of the CRAP will hold (3), we can simplify the objective function. In particular, it is reduced to the following expression:

$$
\begin{aligned}
(n-2) ! & \sum_{i, j \in V-\{1\}: i \neq j} c_{i j}\left(z_{i j}+\sum_{k, t \in V-\{i, j, 1\}: k<t} 0.5+\sum_{k \in V: k \neq i, j, 1} 1\right)+ \\
& \sum_{j \in V-\{1\}} c_{1 j}\left(\sum_{k \in V-\{1, j\}} z_{j k}+\sum_{k, t \in V-\{j, 1\}: k<t} 0.5\right)+ \\
& \left.\sum_{j \in V-\{1\}} c_{j 1}\left(\sum_{k \in V-\{1, j\}} z_{k j}+\sum_{k, t \in V-\{j, 1\}: k<t} 0.5\right)\right)
\end{aligned}
$$

Ignoring the factor $(n-2)$ ! and the constant, those cyclic sequences which minimize the above expression are the same cyclic sequences which minimize

$$
\sum_{i \in V-\{1\}} \sum_{j \in V-\{1\}: j \neq i}\left(c_{i j}+c_{1 i}+c_{j 1}\right) z_{i j} .
$$

Therefore, it is sufficient to consider the objective function given in (7) to obtain the optimal solution of the CRAP.

In order to recover the optimal value of the CRAP, (1), the constant and the factor should also be computed:

$\frac{2 \sum_{s \in R} C(s)}{(n-1) !}-\frac{2}{(n-2)(n-1)^{2}}\left(2 v^{*}+\left(\left(\begin{array}{c}n-3 \\ 2\end{array}\right)+2(n-3)\right) \sum_{i, j \in V-\{1\}: i \neq j} c_{i j}+\left(\begin{array}{c}n-2 \\ 2\end{array}\right) \sum_{j \in V-\{1\}}\left(c_{j 1}+c_{1 j}\right)\right)$

where $v^{*}$ is the optimal value of

$$
\begin{array}{cl}
\left(\mathrm{CRAP}^{*}\right) \max & \sum_{i \in V-\{1\}} \sum_{j \in V-\{1\}: j \neq i}\left(c_{i j}+c_{1 i}+c_{j 1}\right) z_{i j} \\
\text { s.t. } & z_{i j}+z_{j i}=1 \quad \forall i, j \in V: i<j \\
& z_{i j}-z_{i k}-z_{k j} \leq 0 \quad \forall i, j, k \in V: i \neq j, j \neq k, i \neq k \\
& z_{j 1}=1 \quad \forall j \in V-\{1\} \\
& z_{i j} \in\{0,1\} \quad \forall i, j \in V: i \neq j
\end{array}
$$

Even if CRAP* is strongly similar to LOP, the credit of this paper is to prove that the optimal value of a problem which uses the Kendall Tau distances for aggregating cyclic ranks is exactly given by (8). Moreover, since solving the $\mathrm{CRAP}^{*}$ implies solving the LOP, the CRAP is NP-hard. 


\section{The Permutated Asymmetric Traveling Salesman Problem}

Let us consider consider the situation in which the optimal tour given when solving the asymmetric TSP needs to be modified because of unexpected events. We allow the ATSP to admit a source of uncertainty and we wish to hedge against it assuming a robust alternative criterion. If our aim is that tours with large disagreements have fewer probabilities of occurrence than tours with small disagreements we can define the probability that the tour occurs as

$$
p(\rho, r)=\frac{\mathcal{B}(n-1)-d(\rho, r)}{\mathcal{A}(n-1)} \quad \forall \rho, r \in R
$$

where $\mathcal{A}(n)$ is the total number of disagreements in all the permutations of $n$ and $\mathcal{B}(n)$ is the maximum number of disagreements that may occur from any permutation pair of $n$ elements and both values are known to be

$$
\mathcal{A}(n)=n ! n(n-1) / 4, \quad \mathcal{B}(n)=n(n-1) / 2 .
$$

All the probabilities are non negative because $\mathcal{B}(n)$ is the maximum number of possible disagreements, and the addition of any row of the probability matrix is 1 :

$$
\sum_{r \in R} p(\rho, r)=\sum_{r \in R} \frac{\mathcal{B}(n-1)}{\mathcal{A}(n-1)}-\sum_{r \in R} \frac{d(\rho, r)}{\mathcal{A}(n-1)}=(n-1) ! \frac{\mathcal{B}(n-1)}{\mathcal{A}(n-1)}-1=1
$$

In our example, probabilities are those in Table 2, which follow from applying formula $\left(3-\delta_{i j}\right) / 9$ to disagreements in Table 1 .

\begin{tabular}{r|rrrrrr} 
& 1234 & 1243 & 1324 & 1342 & 1423 & 1432 \\
\hline$\rho_{1}=1234$ & $3 / 9$ & $2 / 9$ & $2 / 9$ & $1 / 9$ & $1 / 9$ & 0 \\
$\rho_{2}=1243$ & $2 / 9$ & $3 / 9$ & $1 / 9$ & 0 & $2 / 9$ & $1 / 9$ \\
$\rho_{3}=1324$ & $2 / 9$ & $1 / 9$ & $3 / 9$ & $2 / 9$ & 0 & $1 / 9$ \\
$\rho_{4}=1342$ & $1 / 9$ & 0 & $2 / 9$ & $3 / 9$ & $1 / 9$ & $2 / 9$ \\
$\rho_{5}=1423$ & $1 / 9$ & $2 / 9$ & 0 & $1 / 9$ & $3 / 9$ & $2 / 9$ \\
$\rho_{6}=1432$ & 0 & $1 / 9$ & $1 / 9$ & $2 / 9$ & $2 / 9$ & $3 / 9$
\end{tabular}

Table 2: Probability matrix; Columns are the potential tours and rows are all the possible permutations.

Now, we can define the Permutated Asymmetric Salesman Problems as the problem to find the optimal tour $\rho$ in $R$ for the problem

$$
(\rho \mathrm{ATSP}) \arg \min _{\rho} \sum_{r \in R} C(r) p(\rho, r) .
$$

According to the definition of the probabilities, $\rho$ ATSP is equivalent to CRAP, changing maximization by minimization in the definition of the problems. 


\begin{tabular}{|r|rrrrrr|}
\hline Route & 1234 & 1243 & 1324 & 1342 & 1423 & 1432 \\
\hline$C(\cdot)$ & 5 & 6 & 8 & 9 & 7 & 5 \\
\hline$C^{w}(\cdot)$ & 6.56 & 6.11 & 7 & 7.22 & 6.33 & 6.78 \\
\hline
\end{tabular}

Table 3: Left: Asymmetric cost matrix. Right: Route costs and expected tour costs

$$
\left(\mathrm{CRAP}_{\max }\right) \arg \max _{\rho} \sum_{r \in R} C(r) d(\rho, r)
$$

In fact, if we denote by $v^{*}(\rho A T S P)$ the optimal objective value of $\rho \operatorname{ATSP}, v^{*}(\rho A T S P)=$ $2 \sum_{r \in R} C(r) /(n-1) !-v^{*}\left(C R A P_{\max }\right) / \mathcal{A}(n-1)$.

Table 3 shows the objective value of the ATSP and of the $\rho$ ATSP for all the possible tours for the given cost matrix. In our example, $v^{*}(\rho A T S P)=6.11, \sum_{r \in R} C(r)=40$ and $v^{*}\left(C R A P_{\max }\right)=65$.

It is easy to see that the tour in the reverse direction to an optimal tour for CRAP is an optimal tour for $\mathrm{CRAP}_{\max }$. Given a tour $r \in R$ and the tour in the reverse direction $\bar{r}$ it is always satisfied that

$$
\delta_{r s}+\delta_{\bar{r} s}=\mathcal{B}(n-1)
$$

In Table 1 we can observe that $\delta_{1234, s}+\delta_{1432, s}=3$ for all $s \in\{1234,1243,1324,1342,1423,1432\}$, analogously $\delta_{1243, s}+\delta_{1342, s}=3$ and $\delta_{1324, s}+\delta_{1423, s}=3$ for all $s \in\{1234,1243,1324,1342,1423,1432\}$. Thus, the opposite tour to an optimal tour for CRAP max $_{\text {max }}$ is an optimal tour for CRAP and viceversa. Moreover, $v^{*}(C R A P) / \mathcal{A}(n-1)=v^{*}(\rho A T S P)$.

On the other hand, we would like to emphasize that optimal solutions to the ATSP are not related with optimal solutions to $\rho$ ATSP (see Table 4 where $v^{*}(A T S P)=5$ and $v^{*}(\rho A T S P)=$ 6.11). If we compute the objective value of $\rho$ ATSP for the optimal value of the ATSP it might strongly differ from the optimal value of $\rho$ ATSP. In order to illustrate this fact, we have solved some of the ATSP instances in TSPLIB ([9]) and the results are shown in Table 4: $v$ (ATSP) is the objective value in $\rho$ ATSP for the optimal solution of the ATSP and \%diff stands for

$$
\% \operatorname{diff}=100\left(v(\operatorname{ATSP})-v^{*}(\sigma \operatorname{ATSP})\right) / v^{*}(\sigma \operatorname{ATSP}) .
$$

For the 22 instances presented in the table, the percentage of difference goes from $0 \%$ to $9.82 \%$ (the $0 \%$ corresponds to a special case in which both solutions, the one for the $\sigma$ ATSP and the one for the ATSP coincide). The average difference for the $f t v$ instances is around $6 \%$ and for all the instances presented in the table it is of a $4.8 \%$. These differences indicate that the solutions provided by the ATSP model are not adequate as solutions to the $\rho$ ATSP.

\section{Relationships among the different problems}

In this section we prove that we can obtain the optimal solution of the CRAP from the optimal solution of the LOP or from the optimal solution of the $\rho$ ATSP and viceversa. Since 


\begin{tabular}{lrr}
\hline Instance & $|V|$ & \% Diff \\
\hline ftv33 & 34 & 7.36 \\
ftv35 & 36 & 6.50 \\
ftv38 & 39 & 6.17 \\
ftv44 & 45 & 5.94 \\
ftv55 & 48 & 9.82 \\
ftv64 & 56 & 6.39 \\
ftv70 & 65 & 3.87 \\
ftv90 & 71 & 4.55 \\
ftv100 & 91 & 5.17 \\
ftv110 & 101 & 4.61 \\
ftv120 & 111 & 4.21 \\
ftv130 & 121 & 4.00 \\
ftv140 & 131 & 6.08 \\
ftv150 & 141 & 5.93 \\
ftv160 & 151 & 5.75 \\
ftv170 & 161 & 6.41 \\
br17 & 171 & 5.84 \\
ft53 & 17 & 0.00 \\
ft70 & 53 & 8.41 \\
p43 & 70 & 3.24 \\
ry48p & 43 & 0.11 \\
\hline
\end{tabular}

Table 4: Percentage of deviation for the 22 ATSP instances in the TSPLIB. [9]

LOP and RAP were also proved to be equivalents in [2], we state that LOP, RAP, CRAP and $\rho$ ATSP are equivalent.

\section{Theorem 1}

$L O P, C R A P$ and $\rho A T S P$ are equivalent.

Proof of Theorem 1. Let $L=(V, A)$ be a completed directed graph with arc weight $w_{i j}$ for each pair $i, j \in V$. Let $x_{i j}$ a binary variable which takes the value of 1 iff $i$ goes before $j$ for all $i, j \in V$. The canonical IP formulation of the LOP is:

$$
\begin{array}{ll}
\text { (LOP) } \max & \sum_{(i, j) \in A} w_{i j} x_{i j} \\
\text { s.t. } & x_{i j}+x_{j i}=1 \quad \forall i, j \in V: i<j \\
& x_{i j}-x_{i k}-x_{k j} \leq 0 \quad \forall i, j, k \in V: i \neq j, j \neq k, i \neq k \\
& x_{i j} \in\{0,1\} \quad \forall i, j \in V: i \neq j
\end{array}
$$

- LOP and CRAP are equivalents: Each instance of the LOP can be transformed to an instance of the CRAP* adding an additional null row and null column to the matrix of distances, and each instance of the CRAP* , is by definition an instance of the LOP when 
the arcs weight have been transformed, $w_{i j}=c_{i j}+c_{1 i}+c_{j 1}$ for $i, j \neq 0$, and $w_{i j}=0$ otherwise.

- CRAP and $\rho$ ATSP are equivalent. From the discussion in Section 3, the optimal solution of $\rho$ ATSP is the same cyclic sequence as the optimal solution of CRAP but in the opposite direction.

Theorem 1 implies that the CRAP and the $\rho$ ATSP inherit all the properties of the LOP. For instance that for symmetric weight matrices all the solutions are optimal, among many others.

Since RAP is equivalent to LOP (see [2]), the following Corollary holds.

\section{Corollary 1}

LOP, RAP, CRAP and $\rho A T S P$ are equivalents.

From Corollary 1 it follows that we have introduced two new applications of the LOP devoted to aggregation of preferences and robustness of cyclic sequences.

\section{Acknowledgments}

This work was partly supported by the Spanish Ministry of Economy and Competitiveness through grants MTM2013-46962-C02-01, MTM2012-36163-C06:04, by Regional Government of Andalucía grant FQM5849 and ERDF funds.

\section{References}

[1] A. Andoni, R. Fagin, R. Kumar, M. Patrascu and D. Sivakumar (2008): Efficient similarity search and classification via rank aggregation. In Proceedings of the ACM SIG-MOD International Conference on Management of Data: 1375-1376.

[2] I. Charon and O. Hudry (2007): A survey on the linear ordering problem for weighted or unweighted tournaments. 4OR 5:5-60.

[3] C. Claiborne Price (2009): Applications of Operations Research models to problems in Health Care. University of Maryland.

[4] C. Dwork, R. Kumar, M. Naor and D. Sivakumar (2001): Rank aggregation methods for the web. In Proceedings of the Tenth International World Wide Web Conference (WWW'01): 613-622.

[5] R. Fagin, R. Kumar and D. Sivakumar (2003): Eficient similarity search and classification via rank aggregation. In Proceedings of the ACM SIGMOD International Conference on Management of Data: 301-312. 
[6] D.A. Grundel and D.E. Jeffcoat (2004): Formulation and solution of the target visitation problem. Proceedings of the AIAA 1st Intelligent systems technical conferences.

[7] S. Lrward (2014): Exact solving methods for the target visitation problem. Diploma thesis.

[8] G. Reinelt (1985): The linear ordering problem. Algorithms and applications. Heldermann.

[9] G. Reinelt (1991): TSPLIB - A traveling salesman problem library. ORSA Journal on Computing, 3(4):376-384.

[10] M. Rafael and G.Reinelt (2011): The lineal ordering problem. Exact and heuristic methods in combinational optimization. Springer.

[11] S. Yasutake, K. Hatano, E. Takimoto and M. Takeda (2012): Online Rank Aggregation”. JMLR: Workshop and Conference proceedings 25: 539-553. Asian Conference on Machine Learning. 